\title{
Cranial juvenile psammomatoid ossifying fibroma: case report
}

\author{
Cristina Barrena López, MD, Alicia Bollar Zabala, MD, PhD, and Enrique Úrculo Bareño, MD, PhD \\ Department of Neurosurgery, Pediatric Section, Donostia University Hospital, San Sebastián-Donostia, Guipúzcoa, Spain \\ Juvenile psammomatoid ossifying fibroma (JPOF) is a fibro-osseous tumor that arises in the craniofacial bones in young \\ people. This lesion usually originates in the jaw, orbit, and ethmoid complex but can also be associated with the skull \\ base and calvaria. Diagnosis must be made based on observing typical radiological and histopathological features. \\ Although JPOF is a rare pathological entity, neurosurgeons must consider this odontogenic lesion in the differential diag- \\ nosis of skull masses given the lesion's aggressive behavior and locally invasive growth. Treatment must be gross-total \\ resection. In the following article, the authors present a case of cranial JPOF and discuss various aspects of this entity. \\ http://thejns.org/doi/abs/10.3171/2015.7.PEDS1521
}

KEY WORDS ossifying fibroma; pediatric skull lesions; juvenile psammomatoid ossifying fibroma; oncology

$\mathbf{J}$ UVENILE psammomatoid ossifying fibroma (JPOF) is an uncommon benign pathology, one of many fibroosseous lesions of the craniofacial area. According to the 2005 WHO classification of odontogenic tumors, JPOF is the most common pathology, subclassified as a variant of ossifying fibroma of the craniofacial skeleton with unique features in terms of age at occurrence, location, and clinical and anatomopathological features. ${ }^{1}$

Usually JPOF is seen in children and young adults, arising in the nasal cavity, paranasal sinuses, orbit, or frontoethmoid complex, with a skull base and calvaria location being extremely rare. ${ }^{6}$ Radiological findings can lead to a diagnosis, although in general diagnosis is difficult given the lesion's similarities with other craniofacial fibro-osseous lesion features. On CT studies JPOF can appear as a circumscribed, multiloculated expansile mass with a thick bony wall. Postcontrast MRI may reveal enhancement of this area. Histologically the lesion is characterized by a cellular fibrous stroma and characteristic small, uniform, spherical calcified ossicles resembling psammoma bodies. $^{2-4,6,8}$

Although JPOF is a benign and slow-growing entity, it has local aggressive behavior and can extend into surrounding areas. Thus proper identification of this lesion is important for a definitive diagnosis and therapeutic man- agement. The recommended treatment is gross-total excision since incomplete or partial resection results in a high rate of recurrence.

We present a case of JPOF in the skull bone with a secondary aneurysmal bone cyst and review the tumor's clinical, histopathological, and immunohistochemical aspects and therapeutic management.

\section{Case Report}

History and Examination

A 6-year-old boy with no significant medical history presented with a lesion of the frontoparietal skull on the left side, which had been incidentally discovered by his mother. A consultation was sought at a local hospital, and the patient was referred to our pediatric neurosurgeon for further consultation and management. The mass was painless and hard on palpation. The overlying skin was mobile and normal in appearance without signs of swelling. Physical examination revealed no neurological deficits. Skull radiographs were unclear. A CT scan revealed an expanding intraosseous lytic lesion with a diameter of $2.2 \mathrm{~cm}$. The lesion was well circumscribed, with linear trabeculation and no peripheral sclerosis (Fig. 1). It protruded into the subcutaneous space but had not eroded the inner part of the

ABBREVIATIONS FD = fibrous dysplasia; JOF = juvenile ossifying fibroma; JPOF = juvenile psammomatoid ossifying fibroma; JTOF = juvenile trabecular ossifying fibroma; PEPM = primary extracranial psammomatoid meningioma.

SUBMITTED January 14, 2015. ACCEPTED July 13, 2015.

INCLUDE WHEN CITING Published online November 20, 2015; DOI: 10.3171/2015.7.PEDS1521. 

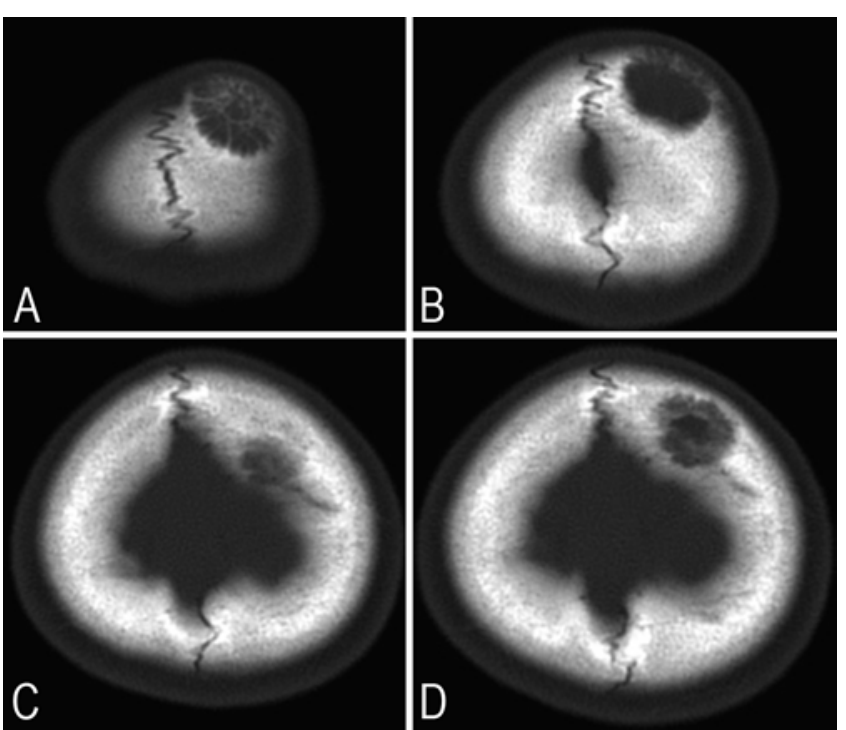

FIG. 1. Axial noncontrast-enhanced CT scans showing an expansive intraosseous lesion. The mass was well circumscribed, with linear trabeculation and no sclerosis.

skull. The lesion exhibited aggressive behavior toward the outer table but not the adjacent structures such as the galea or dura mater. On MRI an intradiploic mass measured 2 $\times 2.3 \times 6.8 \mathrm{~cm}$; it was well demarcated from surrounding bone and displayed an isointense stroma on T1- and T2weighted sequences in relation to the cerebrospinal fluid, with no restricted diffusion on diffusion-weighted imaging and periphery enhancement with gadolinium (Fig. 2).

Observing the clinical and radiographic characteristics, we considered intraosseous cavernous hemangioma to be the most likely diagnosis since the lesion was well demarcated from the galea without surrounding edema and had the expected radiographic findings. Nevertheless extracranial meningioma was another suspected diagnosis in that the lesion had a homogeneous appearance and enhanced after intravenous injection of contrast. The possibility of JPOF was not considered before surgery.

\section{Operation}

Excision was performed via open craniotomy. Because of the localization of the lesion, its dimensions, and our suspected diagnosis, we opted for total removal in 1 piece with at least $1 \mathrm{~cm}$ of security margins. The lesion was ex-
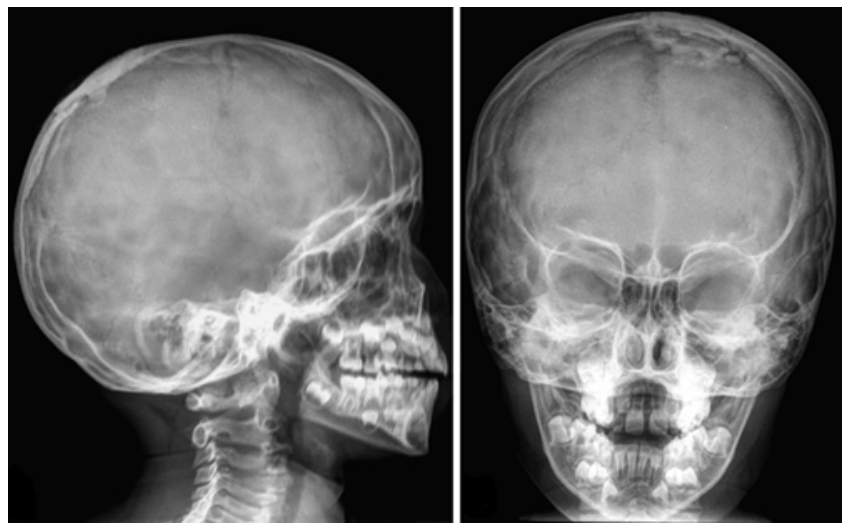

FIG. 3. Postoperative skull radiographs, lateral (left) and anteroposterior (right) views, show the bone reconstruction with material made of tetracalcium phosphate, tricalcium phosphate, and hydroxyapatite with no evidence of tumor recurrence.

cised "en bloc"; the piece was heterogeneous with a translucent blue part suggestive of an aneurysmal cyst. The underlying dura mater was intact. The defect was covered with a bone replacement system. We used a biocompatible material made of tetracalcium phosphate, tricalcium phosphate, and hydroxyapatite.

\section{Postoperative Course}

The patient's postoperative course was uneventful. Clinical and radiological examinations (radiography and MRI) performed 6 months later did not show neurological deficit or recurrence of tumor (Figs. 3 and 4). One year later radiography did not reveal any sign of recurrence, and the patient remained asymptomatic. Nevertheless he will continue to be followed up.

Our Pathological Anatomy Service confirmed the total excision with free edges of tumor (Fig. 5). Histopathological findings consisted of multiple rounded calcified ossicles resembling psammoma bodies embedded in a hypercellular stroma characterized by spindle cells growing in fascicles with focal whorl formations. Some of the ossicles were calcified as in odontogenic fibromas (Fig. 6). No mitotic figures were found. In the periphery the disposition was less cellular with a more fibrous component and a great number of reactive trabecular aspects. There was aneurysmal cystic degeneration. Immunohistochemical analysis was not performed.
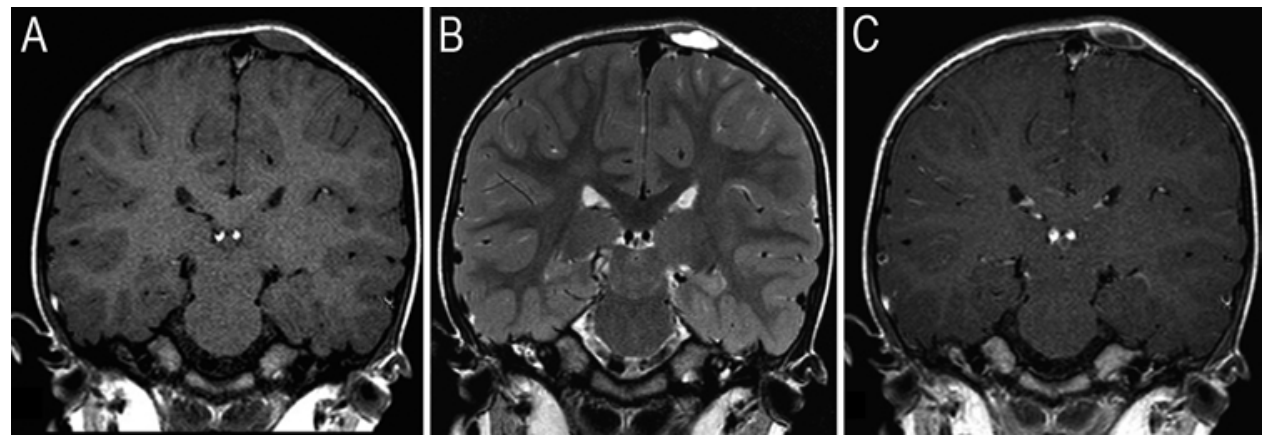

FIG. 2. Cranial coronal MR images. A: T1-weighted imaging shows a circumscribed and homogeneous mass. B: T2-weighted imaging shows 2 different densities. C: T1-weighted imaging with gadolinium showing periphery enhancement. 


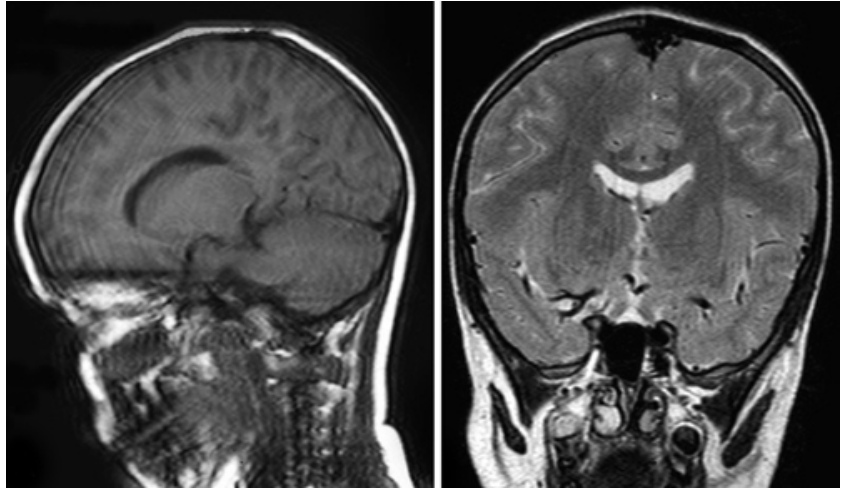

FIG. 4. Postoperative sagittal T1-weighted (left) and coronal T2-weighted (right) MR images confirm complete removal of the lesion.

\section{Discussion}

\section{Terminology Controversy}

"Juvenile psammomatoid ossifying fibroma" has been referred to using different terminology, presenting a nomenclatural controversy between authors and pathologists before its name was settled. The lesion was first reported in 1938 by Benjamins, ${ }^{2}$ who termed it "osteoid fibroma with atypical ossification." In $1949 \mathrm{Gögl}^{7}$ gave it the designation of "psammomatoid ossifying fibroma." The lesion was later named "juvenile active ossifying fibroma" by Johnson $^{11}$ in 1952. Johnson introduced the term "active" based on the aggressive and proliferating behavior of this pathology. Makek ${ }^{16}$ called the lesion "psammous desmoosteoblastoma" as a variant of osteoblastoma. The second edition of the WHO classification of odontogenic tumors used the general term "juvenile ossifying fibroma" (JOF) to describe the lesion affecting the craniofacial skeleton in children under the age of 15 years. ${ }^{14}$ Wenig et al. ${ }^{25}$ who called the lesion "aggressive psammomatoid ossifying fibroma," added the adjective "aggressive" because of the lesion's potential for infiltrative growth and dropped the term "juvenile" because the entity was not confined to the younger age group. Hartstein et al. ${ }^{9}$ were more simple in their description, calling it just "psammomatoid ossifying fibroma" with no allusion to a patient's age or the nature of the lesion. In the 2005 WHO classification of odontogenic tumors, ${ }^{1}$ benign fibro-osseous lesions of the head and neck
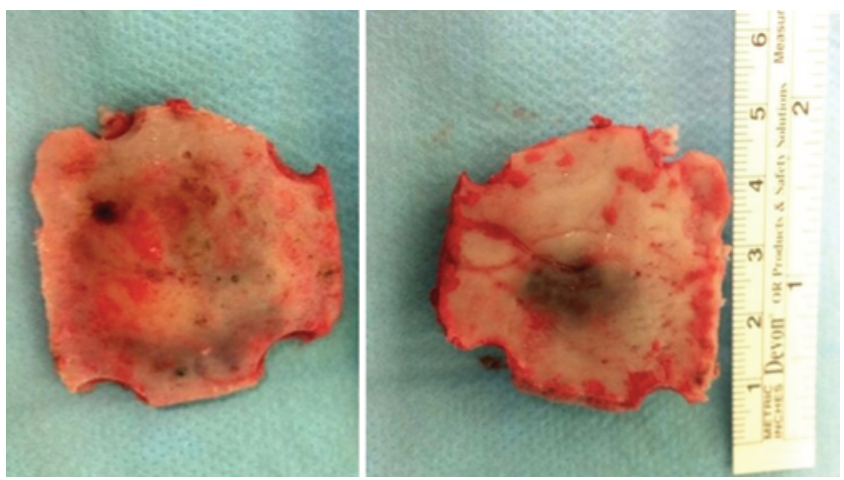

FIG. 5. Macroscopic views of the JPOF. The piece of craniotomy shows the aneurysmal bone cyst. Figure is available in color online only.
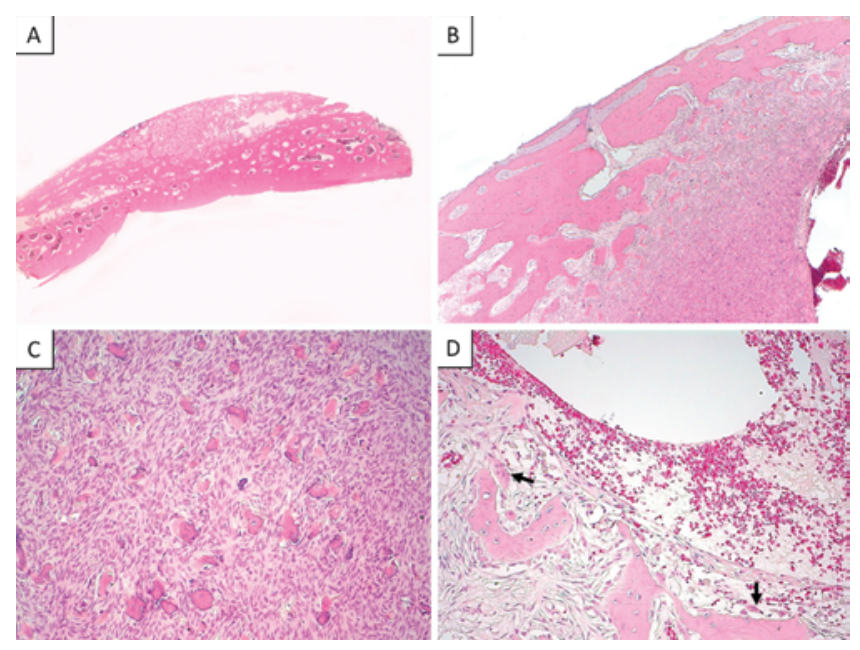

FIG. 6. Histopathological examination of the JPOF showing a panoramic view of the lesion $(A)$. $H \& E$, original magnification $\times 10$. The tumor infiltrates surrounding cortical bone (B). Notice the aneurysmal bone cyst formation (right). $\mathrm{H} \& \mathrm{E}$, original magnification $\times 40$. The lesion $(C)$ is composed of a thin fringe of collagen fibers with hypercellular fusiform stromal cells and numerous homogeneously distributed calcifying ossicles in a concentric fashion (psammoma body-like structures). $\mathrm{H} \& \mathrm{E}$, original magnification $\times 100$. Aneurysmal bone cyst (D) with osteoclastic giant cells (arrows). Figure is available in color online only

are classified as fibrous dysplasia (FD), cemento-osseous dysplasia (COD), and ossifying fibroma (OF). The histopathology in OF involves the replacement of medullar bone by fibrous tissue with a variable quantity of bone. Ossifying fibroma can be subdivided into conventional and juvenile forms (JOF); in turn, JOFs are subdivided into 2 distinct histopathological variants, JPOF and juvenile trabecular ossifying fibroma (JTOF; Table 1). Nowadays JPOF is a lesion with signature pathological features that make it a distinct entity.

\section{Etiology}

Craniofacial fibro-osseous lesions compose a group of disorders characterized by the replacement of bone by a benign connective tissue matrix with varying amounts of mineralized substances. Johnson ${ }^{11}$ suggested that JPOF can arise from junction sutures, which is a site that can produce ossicles and chondroid spheres. Some investigators have hypothesized that JPOF originates from an over-

TABLE 1. WHO histological classification of odontogenic tumors

\begin{tabular}{c}
\hline Ossifying fibroma \\
\hline Conventional ossifying fibroma \\
\hline Juvenile ossifying fibroma \\
Juvenile psammomatoid ossifying fibroma \\
Juvenile trabecular ossifying fibroma \\
\hline Fibrous dysplasia \\
\hline Osseous dysplasia \\
\hline Central giant cell lesion (granuloma) \\
\hline Cherubism \\
\hline Aneurysmal bone cyst \\
\hline Simple bone cyst
\end{tabular}


production of the myxofibrous cellular stroma. These stromal cells secrete hyaline material that can ossify.

Recently, a new tumor suppressor gene (HRPT2) mutation has been related to ossifying fibroma, and it has been suggested that such lesions could arise as a result of the haploinsufficiency of that particular gene. ${ }^{20}$

\section{Clinical Features}

Single case reports as well as several smaller series have appeared in the literature. The major series reported by Johnson et al. ${ }^{12}$ and Makek, ${ }^{16}$ with 112 and 86 cases, respectively, showed findings similar to JPOF. The majority of cases occurred in patients with an average age from $10-15$ to $25-30$ years, although a considerable number of cases referred to older patients. The entity is exceptional in very young patients; nevertheless, our case of JPOF occurred in a 6-year-old male child. A slight male predominance has been observed. The most common lesion sites were the paranasal sinuses and the jaw (70\% in the paranasal sinuses, followed by $20 \%$ in the maxilla and $10 \%$ in the mandible), and just $10 \%$ occurred in the calvaria. In the mandible, JPOF used to occur more in the ramus than in the body of the mandible. The majority of single cases documented thus far have affected the sinonasal bones too, with a few occurring in the neurocranium. In the calvaria location, JPOF has been reported only rarely, with the anterior cranial fossa as the most frequent location. ${ }^{3}$ As regards skull bones, single cases have been documented in the parietal, temporal, and frontal bones. ${ }^{5}$ Normally JPOF progresses in an asymptomatic way with no clinical neurological deficits, but it depends on the lesion's location. As in the present case, calvarial JPOF has been discovered accidentally following a minor head injury or in the course of a diagnostic workup. In the facial area, the most commonly observed clinical manifestation has been proptosis. ${ }^{16}$ Lateral displacement of the eyeball and decreasing vision have also been reported. If the lesion reaches the paranasal sinus, nasal obstruction, recurrent sinusitis, pain, and facial swelling may be other clinical findings. If the lesion occurs in the mandible, the symptoms often consist of painless local swelling. Juvenile psammomatoid ossifying fibroma grows slowly, although it is characterized by its invasive growth. Extension to the adjacent craniofacial cavities may vary from just pushing the adjacent bone to invasive growth and bone erosion of the nearby anatomical structures. ${ }^{22}$

Aggressive growth has been observed in some cases, mainly for lesions in a maxillary location and in younger patients in the 1st and 2nd decades of life. ${ }^{12}$ This behavior has been associated with aneurysmal bone cyst formation. The development of aneurysmal bone cyst seems to be a common transformation and is documented in many cases, as in our case..$^{16}$ The etiology and the predictive factors of cystic transformation remain unclear, but they may be related to genetic and/or environmental causes. ${ }^{10}$ Clinically cyst formation has been the cause of accelerated tumor growth in a short period of time, although patients remain pain free and with no neurological deficit. The cyst occurs more commonly in children.

Despite the tumor's aggressive growth and recurrence rate, malignant transformation or cases of metastasis have not been reported until now. Complications due to direct intracranial extension with secondary encephalitis and meningitis have been reported. ${ }^{12}$

\section{Imaging Characteristic}

The radiological diagnosis of JPOF is suggested by the findings on CT with bone windows and MRI; however, radiological features can resemble other odontogenic lesions. In radiology, JPOF may show areas of "ground glass" with a lesion not encapsulated but separated with radiopaque borders. ${ }^{18}$ Computed tomography studies show an expansive but circumscribed lesion with an abrupt transition of thick bone at the margin of the lesion. The internal appearance may be multiloculated. In CT bone windows, abnormal bone can be differentiated from the rest of the adjacent bony structures. Magnetic resonance imaging shows intensities similar to the gray matter of the brain on precontrast T1-weighted images and low intensities on T2-weighted sequences. Gadolinium contrast MRI may show enhancement of the thick outer extradural lesion. In the mandible the lesion may appear circumscribed and expansive with calcified matrices. In the sinonasal area the lesion can protrude into an air-filled space, keeping the margin clearly demarcated, although in this area the lesion could show a disrupted margin surrounding the bony structure.

\section{Histopathology}

Juvenile psammomatoid ossifying fibroma is one of the benign fibro-osseous lesions that belong to a diverse group of conditions with common histopathological features. These lesions may show hypercellular fibroblastic vascular stroma, with a variety of calcified matrices represented by woven bone and lamellar bone, curvilinear trabeculae, and calcifications. However, JPOF is characterized by the presence of multiple round, uniform small masses of osteoid with calcifications in a concentric pattern, imparting a psammoma body-like appearance. These ossicles can resemble dental cementum but may have an osteoid rim. The nonosseous component can vary from densely cellular to loose, with cellular proliferation of uniform fibroblastic spindle cells and multinucleate osteoclast-like giant cells. ${ }^{22}$ Mitotic figures are extremely rare, and neither atypia nor necrosis is identified. As discussed earlier in this report, the development of aneurysmal bone cyst in JPOF has been reported. It begins with focal myxoid changes and microcystic spaces in the stroma, and gradual expansion of the cyst can form a thin fibrous wall. Aggregates of osteoclast-like giant cells or hemorrhage may appear in association with cystic change.

Immunohistochemistry is not routinely performed to diagnose. Stromal cells of JPOF usually express vimentin, epithelial membrane antigen (EMA), smooth muscle actin (SMA) and CD10, with a lack of expression of CD34, S100 protein, and cytokeratins. ${ }^{8}$ The proliferation index (Ki 67) is frequently low and not useful to predict aggressive behavior. ${ }^{10}$ Little is known about the aggressive biological behavior of this lesion.

\section{Differential Diagnostic}

The identification of JPOF as a distinct type of odon- 
togenic lesion is important considering the potential for local aggressive behavior, and in children the differential diagnosis of a skull tumor differs from that in the adult population. Juvenile psammomatoid ossifying fibroma and FD are 2 fibro-osseous lesions with overlapping features but with different disease progression. In contrast to JPOF, FD is a poorly circumscribed bony expansion not encapsulated by a thin cortex with various degrees of radiopacity on neuroimaging studies ${ }^{28}$ Histopathological characteristics of FD consist of cellular fibrous tissue with spindle-shaped cells with irregularly shaped trabeculae of woven bone with no osteoblastic rimming. ${ }^{7}$ Fibrous dysplasia has been linked to genetic alterations of GNAS1.

The 2 types of JOF-JPOF and JTOF-are easily differentiated by their histopathological features and the patient's condition. ${ }^{12}$ Juvenile trabecular ossifying fibroma occurs in younger patients than $\mathrm{JPOF}^{17}$ and usually arises in the craniofacial bones, especially around the paranasal sinuses and orbits. Juvenile trabecular ossifying fibroma is characterized by progressive and rapid growth leading to facial asymmetry. On neuroimaging JTOF is a welldefined unilocular or multilocular radiolucent mass with cortical thinning and perforation. Microscopic examination of JTOF contains a cell-rich fibroblastic spindle cell stroma, osteoid matrix, and trabeculae of immature woven bone. ${ }^{23}$ Progressive calcification of the osteoid results in anastomosing trabeculae of immature woven bone.

Juvenile psammomatoid ossifying fibroma may be confused with cementum-forming neoplasms, such as cementoblastoma or cementifying fibroma. However this kind of tumor occurs frequently in the 3rd and 4th decades and develops in the mandibular premolar-molar area. These lesions produce "cementum-like" material, structures called "droplets" or "cementicles," which show oval mineralized spherules. ${ }^{10}$

The clinical differential diagnosis includes primary extracranial psammomatoid meningioma (PEPM). Juvenile psammomatoid ossifying fibroma may be difficult to distinguish from PEPM given the macroscopic anatomical superficial similarity, neurocranial location, and radiological aspects. Primary extracranial psammomatoid meningioma is an uncommon pathology, just $2 \%$ of all meningiomas. They may arise from heterotopic rests of arachnoid cells in locations such as the nasal cavity, paranasal sinuses, and nasopharyn $\mathrm{x}^{24}$ and show an irregular bone surface. Histopathologically in psammomatoid meningioma there is a random distribution of psammoma bodies, which are rimmed by a group of osteoclasts and osteoblasts. Psammomatoid meningioma is immunoreactive for epithelial membrane antigen and vimentin, a differential characteristic of PEPM. However most meningiomas show meningothelial cells that react positive for antibodies directed against S100 protein, and CD10 is expressed in a high percentage of PEPMs. ${ }^{8}$

Benign skull base lesions in children such as primary intraosseous cavernous hemangioma or eosinophilic granuloma have to be considered. Cavernous hemangiomas of the skull are extremely rare tumors usually established in frontal and parietal bones. As they enlarge, they may cause periodic severe headaches. On CT they demonstrate reactive osteoclastic and osteoblastic remodeling, with the characteristic trabeculated honeycomb configurations. Microscopically, intraosseous cavernous hemangiomas are composed of thin-walled vascular channels lined by a single layer of flattened endothelial cells interspersed among bony trabeculae. ${ }^{19}$ Eosinophilic granuloma, a variety of histiocytosis $X$, is a benign disorder that affects children and young adults too. The clinical and radiographic findings are often not specific enough to determine the diagnosis, but the key feature is the identification of Langerhans cells. ${ }^{13}$

\section{Therapeutic Management}

The recommended treatment must be complete excision of the tumor. Recurrence after partial or incomplete resection is common, ranging from $30 \%$ to $56 \% .^{1-4,7}$ Lesions with significant cortical destruction and periosteal elevation have an increased risk of recurrence. Resection requires tumor-free margins for the preservation of important adjacent structures as much as possible. Recurrence can depend on the difficulty of excision in certain locations and the infiltrative tumor borders. In a cranium location, open surgical management is strongly preferred inasmuch as it allows greater visibility and exposure of the tumor and adjacent areas of extension (ethmoid and frontal sinuses, orbit, and so forth); however, an endoscopic endonasal approach has been used alone or in combination with a second open surgery. ${ }^{21}$ At the time of tumor excision, reconstruction must be performed if possible. Surgical complications may appear as blood loss requiring transfusion or in cases of tumor associated with the anterior cranial base, or secondary complications can occur as a direct result of the destruction of vital structures. ${ }^{18}$ For JPOF in the calvaria, bone grafts or other osteogenic materials can be used. For large tumors of the anterior cranial fossa, the skull defect can be reconstructed with a periosteal pedicle and an absorbable mesh to reduce comorbidity and improve survival. ${ }^{15}$ Regardless of the approach, close clinical and radiological follow-up is essential to detect and treat recurrences. In cases of subtotal resection, adjuvant treatment is not indicated. Radiotherapy is contraindicated due to the radioresistance of JPOF, and chemotherapy is not necessary in this kind of tumor. ${ }^{22}$ Clinical guidelines of follow-up protocols have not yet been standardized in the literature, but re-excision should be considered. Recurrence can be observed after a period ranging from 6 months to 19 years. ${ }^{23}$

\section{Conclusions}

Juvenile psammomatoid ossifying fibroma is a variety of ossifying fibroma with unique histopathological features that configure its identity. The lesion commonly affects young people, with a predilection for the jaw and sinonasal region. A calvarial location is rare, but neurosurgeons must consider this entity in the differential diagnoses of skull lesions because its aggressive growth and local invasion need special surgical considerations and close clinical and radiological follow-up. On neuroimaging JPOF can appear as a circumscribed lesion with a degree of calcifications and cystic changes. Histologically its signature characteristic is a cellular fibrous stroma with 
spheroid calcifications such as psammoma bodies. Treatment must be complete excision with free margins. Despite the partial or incomplete resection results in recurrences, JPOF has a good prognosis.

\section{Acknowledgments}

We thank the Department of Pathology of Donostia University Hospital for support and providing us with access to the pictures of this pathology. We also appreciate the comments about Fig. 6 .

\section{References}

1. Barnes L, Everson J, Reichart P, Sidransky D (eds): World Health Organization Classification of Tumours: Pathology and Genetics of Head and Neck Tumours. Lyon, France: IARC Press, Vol 9, 2005

2. Benjamins CE: Das osteoid-fibroma mit atypischer Verkalkung im Sinus frontalis. Acta Otolaryngol 26:26-34, 1938

3. Bertrand B, Eloy P, Cornelis JP, Gosseye S, Clotuche J, Gilliard C: Juvenile aggressive cemento-ossifying fibroma: case report and review of the literature. Laryngoscope 103:13851390,1993

4. Damjanov I, Maenza RM, Snyder GG III, Ruiz JW, Toomey JM: Juvenile ossifying fibroma: an ultrastructural study. Cancer 42:2668-2674, 1978

5. El-Mofty S: Psammomatoid and trabecular juvenile ossifying fibroma of the craniofacial skeleton: two distinct clinicopathologic entities. Oral Surg Oral Med Oral Pathol Oral Radiol Endod 93:296-304, 2002

6. El-Mofty S, Kyriakos M: Psammomatoid ossifying fibromas: immnunohistochemical analysis and differential diagnosis with psammomatous meningiomas of cranial bones, in Gnepp DR (ed): Diagnostic Surgical Pathology of the Head and Neck. Philadelphia: Saunders, 2001

7. Gögl H: Das psammo-osteoid fibroma der nase und ihrer Nebenhölen. Monatsschr Ohrenheilkd Laryngorhinol 83:1-10, 1949

8. Granados R, Carrillo R, Nájera L, García-Villanueva M, Patrón M: Psammomatoid ossifying fibromas: immunohistochemical analysis and differential diagnosis with psammomatous meningiomas of craniofacial bones. Oral Surg Oral Med Oral Pathol Oral Radiol Endod 101:614-619, 2006

9. Hartstein ME, Grove AS Jr, Woog JJ, Shore JW, Joseph MP: The multidisciplinary management of psammomatoid ossifying fibroma of the orbit. Ophthalmology 105:591-595, 1998

10. Hasselblatt M, Jundt G, Greiner C, Rama B, Schmäl F, Iglesias-Rozas JR, et al: Juvenile psammomatoid ossifying fibroma of the neurocranium. Report of four cases. J Neurosurg 102:1151-1154, 2005

11. Johnson LC: Bone pathology seminar. American Academy of Oral Pathology 1952:13, 1952

12. Johnson LC, Yousefi M, Vinh TN, Heffner DK, Hyams VJ, Hartman KS: Juvenile active ossifying fibroma. Its nature, dynamics and origin. Acta Otolaryngol Suppl 488:1-40, 1991

13. Kaul R, Gupta N, Gupta S, Gupta M: Eosinophilic granuloma of skull bone. J Cytol 26:156-157, 2009
14. Kramer IR, Pindborg JJ, Shear M: Histological Typing of Odontogenic Tumours, ed 2. Berlin: Springer, 1992

15. Lund VJ, Howard DJ, Wei WI, Cheesman AD: Craniofacial resection for tumors of the nasal cavity and paranasal sinuses-a 17-year experience. Head Neck 20:97-105, 1998

16. Makek M (ed): Clinical Pathology of Fibro-Osteo-Cemental Lesions of the Cranio-Facial Skeleton and Jaw Bones. Basel, Switzerland: Karger:128-227, 1983

17. Margo CE, Ragsdale BD, Perman KI, Zimmerman LE, Sweet DE: Psammomatoid (juvenile) ossifying fibroma of the orbit. Ophthalmology 92:150-159, 1985

18. Noffke CE: Juvenile ossifying fibroma of the mandible. An 8 year radiological follow-up. Dentomaxillofac Radiol 27:363-366, 1998

19. Peterson DL, Murk SE, Story JL: Multifocal cavernous hemangioma of the skull: report of a case and review of the literature. Neurosurgery 30:778-782, 1992

20. Pimenta FJ, Gontijo Silveira LF, Tavares GC, Silva AC, Perdigão PF, Castro WH, et al: HRPT2 gene alterations in ossifying fibroma of the jaws. Oral Oncol 42:735-739, 2006

21. Rowland NC, Jermakowicz WJ, Tihan T, El-Sayed IH, McDermott MW: Subacute cystic expansion of intracranial juvenile psammomatoid ossifying fibroma. J Neurosurg Pediatr 11:687-691, 2013

22. Sarode SC, Sarode GS, Waknis P, Patil A, Jashika M: Juvenile psammomatoid ossifying fibroma: a review. Oral Oncol 47:1110-1116, 2011

23. Slootweg PJ, Panders AK, Koopmans R, Nikkels PG: Juvenile ossifying fibroma. An analysis of 33 cases with emphasis on histopathological aspects. J Oral Pathol Med 23:385388, 1994

24. Swain RE Jr, Kingdom TT, DelGaudio JM, Muller S, Grist WJ: Meningiomas of the paranasal sinuses. Am J Rhinol 15:27-30, 2001

25. Wenig BM, Vinh TN, Smirniotopoulos JG, Fowler CB, Houston GD, Heffner DK: Aggressive psammomatoid ossifying fibromas of the sinonasal region: a clinicopathologic study of a distinct group of fibro-osseous lesions. Cancer 76:11551165,1995

\section{Disclosures}

The authors report no conflict of interest concerning the materials or methods used in this study or the findings specified in this paper.

\section{Author Contributions}

Conception and design: Barrena López. Acquisition of data: Barrena López, Bollar Zabala. Analysis and interpretation of data: Barrena López. Drafting the article: Barrena López. Critically revising the article: Bollar Zabala. Reviewed submitted version of manuscript: Bollar Zabala, Úrculo Bareño. Approved the final version of the manuscript on behalf of all authors: Barrena López. Administrative/technical/material support: Barrena López. Study supervision: Barrena López.

\section{Correspondence}

Cristina Barrena López, Reina Sofia St. 103, Alpera (Albacete) 02690, Spain.email: barrenika@gmail.com. 